2009-01-01

\title{
Antenna Optimization with a Computationally Efficient Multiobjective Evolutionary Algorithm
}

Matthias John

Technological University Dublin, matthias.john@tudublin.ie

Max Ammann

Technological University Dublin, max.ammann@tudublin.ie

Follow this and additional works at: https://arrow.tudublin.ie/engscheceart

Part of the Electrical and Computer Engineering Commons

\section{Recommended Citation}

John, M. \& Ammann, M. (2009)Antenna optimization with a computationally efficient multiobjective evolutionary algorithm. IEEE Transactions on Antennas and Propagation, Vol. 57, no. 1, pp.260-263. doi10.1109/TAP.2008.2009775

This Article is brought to you for free and open access by the School of Electrical and Electronic Engineering at ARROW@TU Dublin. It has been accepted for inclusion in Articles by an authorized administrator of ARROW@TU Dublin. For more information, please contact arrow.admin@tudublin.ie, aisling.coyne@tudublin.ie, gerard.connolly@tudublin.ie.

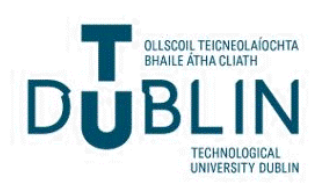


designed antenna consists of three pairs of split-ring loops and a tapered transmission line. The simulated and measured results show that the proposed UWB antenna has a wide bandwidth from 2 to $20 \mathrm{GHz}$, and all the measured return losses are less than $-10 \mathrm{~dB}$ in this band. The graph of the magnitude of the transfer function is relatively smooth combined with a flat group delay in the measured band. The simple planar geometry also makes it compatible with the existing microwave integrated circuit.

\section{REFERENCES}

[1] K. L. Wong, Compact and Broad-Band Microstrip Antennas. New York: Wiley, 2002.

[2] D. Kwon and Y. Kim, "Suppression of cable leakage current for edge-fed printed dipole UWB antennas using leakage-blocking slots," IEEE Antennas Propag. Lett., vol. 5, pp. 183-186, 2006.

[3] Z. N. Chen, "Novel bi-arm rolled monopole for UWB applications," IEEE Trans. Antennas Propag., vol. 53, no. 2, pp. 672-677, Feb. 2005.

[4] J. Liang, C. Chiau, X. Chen, and C. G. Parini, "Study of a printed circular disc monopole antenna for UWB systems," IEEE Trans. Antennas Propag., vol. 53, no. 11, pp. 3500-3504, Nov. 2005.

[5] J. Liang, L. Guo, C. C. Chiau, X. Chen, and C. G. Parini, "Study of CPW-fed circular disc monopole antenna," IEE Proc. Microw., Antennas Propag., vol. 152, no. 6, pp. 520-526, Dec. 2005.

[6] Q. Wu, R. H. Jin, J. P. Geng, and M. Ding, "CPW-fed quasi circular monopole with very wide bandwidth," Electron. Lett., vol. 43, no. 2, pp. 69-70, Feb. 2007.

[7] M. Ding, R. H. Jin, J. P. Geng, and Q. Wu, "Design of a CPW-fed ultrawide band fractal antenna," Microw. Opt. Technol. Lett., vol. 49, no. 1, pp. 173-176, Jan. 2007.

[8] N. Behdad and K. Sarabandi, "A multiresonant single-element wideband slot antennas," IEEE Antennas Propag. Lett., vol. 3, pp. 5-8, 2004.

[9] K. Chang, Microwave Ring Circuits and Antennas. New York: Wiley, 1996.

[10] J. S. Chen, "Dual-frequency annular-ring slot antennas fed by CPW feed and microstrip line feed," IEEE Trans. Antennas Propag., vol. 53, no. 1, pp. 569-571, Jan. 2005.

\section{Antenna Optimization With a Computationally Efficient Multiobjective Evolutionary Algorithm}

\author{
Matthias John and Max J. Ammann
}

Abstract-An efficient multiobjective evolutionary algorithm is described for optimizing a novel spline based printed monopole antenna. The antenna geometry is based on spline outlines. Both radiating element and groundplane are simultaneously optimized by the algorithm. The resulting antenna performance is evaluated. It is shown that the evolutionary algorithm and the spline geometry can be used to efficiently generate ultrawideband antennas on limited computing resources.

Index Terms-Antenna optimization, multiobjective evolutionary algorithm, ultrawideband (UWB) antenna, spline.

\section{INTRODUCTION}

Evolutionary optimization methods such as genetic algorithms (GAs) and particle swarm optimization (PSO) have successfully been used to solve electromagnetic problems. These techniques have received great attention because they can solve a variety of problems and are easy to implement.

These algorithms start with solutions placed randomly in the search space and then evolve towards an optimum. Goodness of the solutions is assessed by a fitness function. This fitness function is usually computed from the results of a commercial or custom built electromagnetic solver.

The time these solvers need to compute the fitness of one trial solution varies from a few seconds to tens of minutes. The optimization algorithm has to trigger the solver hundreds or thousands of times during its run. For example, GAs have been reported [1]-[5] which run populations of 20-60 over 40-600 generations; thus needing up to 30000 function evaluations. Reported PSOs [6]-[8] evolve swarms of 10-800 for 1000-10000 iterations. The maximum number of times the EM solver is run in the cited papers is 200000 . The total number of evaluations has to be weighted against the time per evaluation needed by the solver. Also, parallelization and the number of available computers have to be taken into consideration when choosing the setup for the algorithms.

This paper adopts an efficient global optimizer (EGO) reported by Joshua Knowles in [9]. This algorithm is designed to solve multiobjective optimization problems where each function evaluation is temporally or otherwise expensive. With the time taken by an EM solver being typically in the order of minutes, electromagnetic problems are ideal candidates for this optimization algorithm. The algorithm is suited for scenarios where computational power is limited or only a single license for commercial electromagnetic code is available, and thus, parallelization is not an option. The cited paper [9] compares the ParEGO to different GAs on a number of different theoretic problems, illustrating the performance benefits of ParEGO.

The algorithm selects the trial solution which is most likely to improve the best fitness before evaluation with the time expensive EM

Manuscript received November 21, 2007; revised July 03, 2008. Current version published March 04, 2009.

The authors are with the Centre for Telecommunications Value-chain Research, School of Electronic and Communications Engineering, Dublin Institute of Technology, Dublin 8, Ireland (e-mail: ammann@ieee.org).

Color versions of one or more of the figures in this comment are available online at http://ieeexplore.ieee.org.

Digital Object Identifier 10.1109/TAP.2008.2009775 


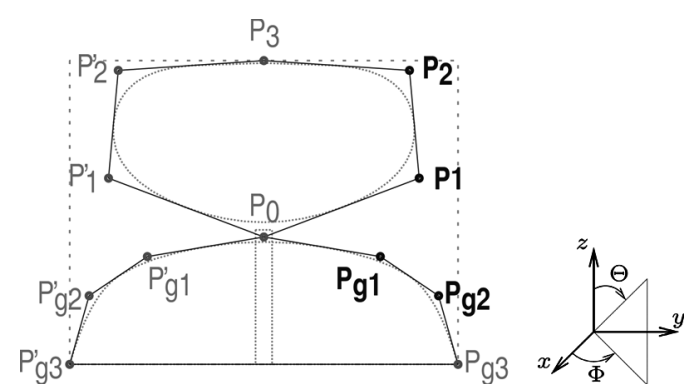

Fig. 1. Spline based geometry with control points.

solver. In order to do that, the algorithm creates a model of the search landscape. This model is updated after every evaluation.

The algorithm itself is more complex than a GA or PSO but it is able to solve the problem in a relatively small number of evaluations, which is fixed at 100 or 250 in the cited paper [9]. In this study, 150 evaluations were sufficient to solve the given task.

In this paper we use the ParEGO algorithm to optimize a spline based printed monopole antenna [10]. Both the radiating element and the groundplane are optimized simultaneously. The ParEGO will run for 150 iterations on a single computer. The performance of the generated ultrawideband (UWB) antenna will be evaluated.

\section{SPline BASED ANTENNA GeOMETRY}

The geometry of the antenna is constructed using quadratic Bézier spline curves. The use of the spline shapes for both radiator and groundplane leads to a less constrained geometry, with a greater freedom for the optimization and can provide resultant forms with less corners/edges and improved antenna performance. The principle of this spline based geometry is shown in Fig. 1. The radiating element of the printed monopole uses 6 control points. Two of these points are fixed, one where the element is fed $\mathrm{P} 0(0 \mathrm{~mm}, 0 \mathrm{~mm})$ and one at the top of the element P3 (0 mm, $18 \mathrm{~mm})$. Points P'1 and P'2 are mirrored copies of points $\mathrm{P} 1$ and $\mathrm{P} 2$. This is to keep the radiating element symmetrical.

The groundplane on the back of the substrate is also constructed by a spline curve. The lower edge of the GP is a straight line; the spline starts at $\mathrm{Pg} 3$ and ends at P'g3 which are fixed. P0 at the top of the GP is also fixed. Points P'g1 and P'g2 are mirrored copies of points Pg1 and $\mathrm{Pg} 2$. The GP is also symmetrical in the $\mathrm{z}$ axis.

The geometry of the antenna has been chosen in a way it needs only a small number of parameters to give great freedom in the shapes achievable by varying these parameters. The geometry is effectively controlled by moving the four points $\mathrm{P} 1, \mathrm{P} 2, \mathrm{Pg} 1$ and $\mathrm{Pg} 2$. Their $\mathrm{z}$ and $y$ coordinates present the eight parameters of the evolutionary optimization algorithm. Limiting the number of parameters to eight also aids the performance of the optimization algorithm. The antenna is printed on double sided Rogers RO4350B substrate of $\varepsilon_{\mathrm{r}}=3.48$ and $\tan \delta=0.0031$. The copper cladding is $17 \mu \mathrm{m}$ thick.

\section{EFFICIENT GLOBAL OPTIMIZATION ALGORITHM}

Recently Knowles presented an evolutionary global optimization algorithm for expensive multiobjective cost functions [9]. The ParEGO algorithm is based on the design and analysis of computer experiments (DACE) model. An initial number of solutions are generated in a Latin hypercube and evaluated with the expensive cost function using CST Microwave Studio (MWS). The initial DACE model is then generated to explain these solutions.

In the optimization phase the algorithm tries to predict a trial solution which is most likely to improve the best fitness found so far. In order to

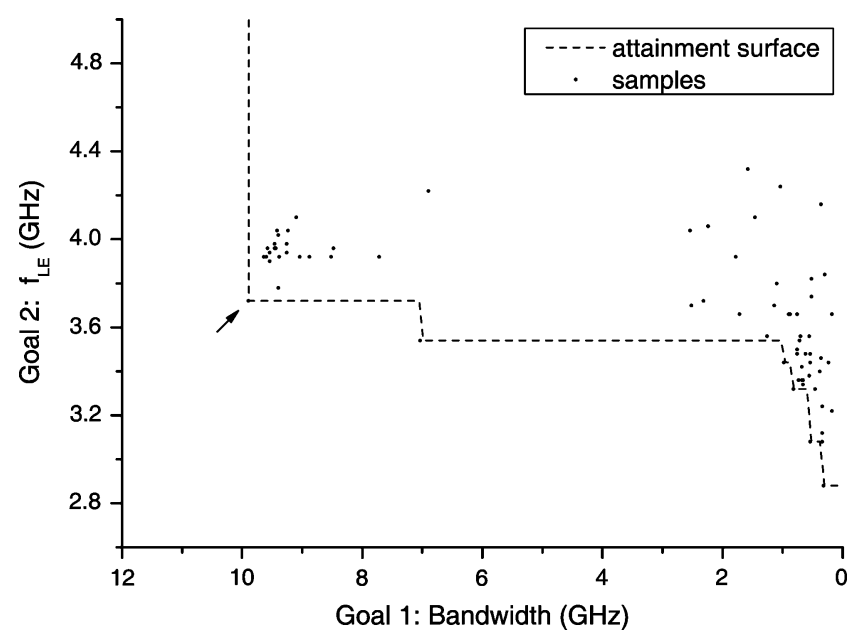

Fig. 2. Attainment surface of the ParEGO.

do that, a GA is embedded which searches the model for this new trial solution. This GA has a population size of 20 and evolves over 10000 generations. This trial solution is then evaluated using the expensive EM solver. The DACE model is then updated and the next iteration starts.

With each iteration, the complexity of the model grows and algorithm takes longer to update the model and to find the next solution. The algorithm is stopped after 150 function evaluations.

In order to solve multiobjective problems, the algorithm updates the weighting between the objectives for every iteration, thus gradually building up the whole Pareto front. The ParEGO is implemented as a minimizer.

For the optimization of the spline based geometry, two objectives are set up which define the performance of the antenna. The first objective is a $-15 \mathrm{~dB}$ return loss bandwidth, all frequency samples where the return loss exceeds $-15 \mathrm{~dB}$ are summed up. Since the algorithm is implemented as a minimizer the sum has to be subtracted from the possible maximum $(N)$.

The first objective function is $N-\sum_{n=0}^{N} f_{n}\left(S^{11}<-15 \mathrm{~dB}\right)$, where $f_{n}$ is the $n$th frequency point from the simulation and $N$ is the number of frequency points used in the simulation $(N=1000$, $0 \leq n \leq N)$.

The second objective is the Lower edge frequency $f_{L E}$. This objective is defined by the first frequency point that exceeds $-15 \mathrm{~dB}$ return loss.

The algorithm was used as it is presented in [9], only the setup function which defines the range and number of parameters and objectives has been adopted. It ran on a single P4 $2.8 \mathrm{GHz}$ PC with 1GB RAM. A single evaluation of a trial solution in CST Microwave Studio takes about 12 min.

The ParEGO was set to run MWS only 150 times. It takes a considerable amount of time to perform the matrix operations needed to keep the model up to date and select the next solution for evaluation. As the optimization progresses, the model of the search landscape grows and the computations take longer. The first update of the model at the 87th iteration took about $4 \mathrm{~min}$ while the last update at the 150th iteration took $23 \mathrm{~min}$. The overall time needed by the optimization amounts to $51.5 \mathrm{~h}$. This gives an average time per iteration of $20.6 \mathrm{~min}$.

From the results of one run of the algorithm, an attainment surface is computed. Fig. 2 shows the attainment surface computed from the output of the algorithm. Each point represents one solution, with the two objectives plotted on the axes. The dashed line is the best attainment surface. Moving along this line, it is not possible to improve one 


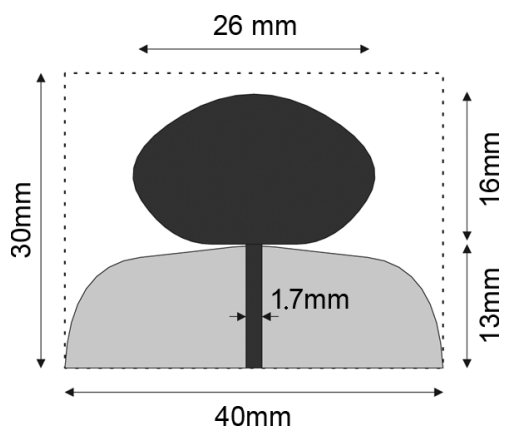

Fig. 3. Antenna design generated by the ParEGO algorithm.

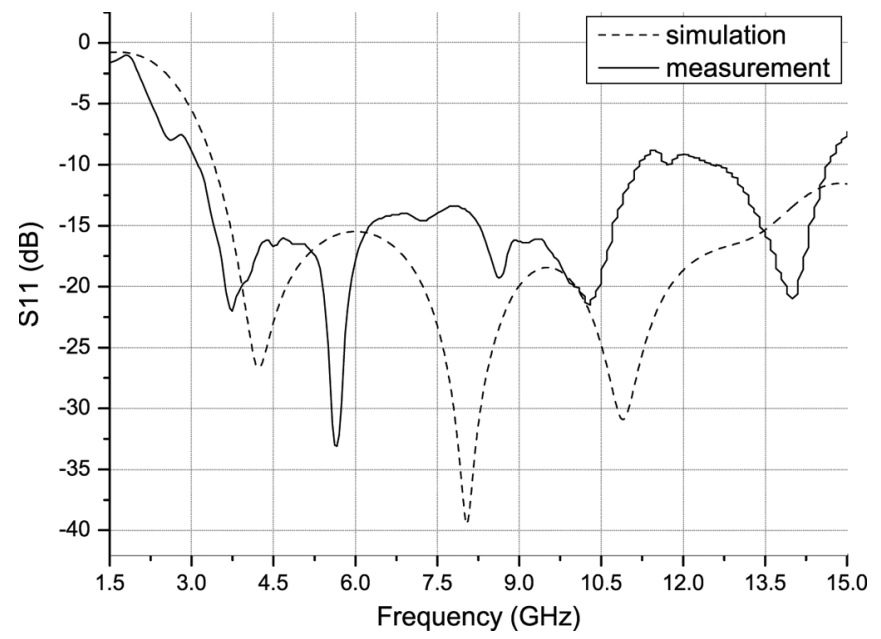

Fig. 4. Simulated and measured return loss for the optimized antenna.

goal without sacrificing performance in the other. From this line a suitable solution can be picked for a required bandwidth or lower edge frequency.

The solution picked for evaluation is marked by the arrow in Fig. 2. It has a $-15 \mathrm{~dB}$ return loss bandwidth of $9.9 \mathrm{GHz}$ starting at $3.7 \mathrm{GHz}$.

The algorithm was run an additional 10 times to verify the robustness of the algorithm. Suitable solutions for UWB antennas which meet the bandwidth and lower edge frequency requirements were repeatedly found.

\section{RESULTS}

The algorithm proved successful in finding suitable positions for the spline control points. The resulting geometry is shown in Fig. 3. It can be seen, that both the radiating element as well as the ground plane curve smoothly away from the feed point. Thus, ensuring good match over a large bandwidth.

The simulated and measured return loss of the antenna is shown in Fig. 4. The resulting antenna geometry is suitable for UWB applications. The simulated bandwidth of the antenna generated by the EGO is from 3.7-13.6 GHz, considering the $-15 \mathrm{~dB}$ goal for the return loss. The measured $-10 \mathrm{~dB}$ return loss bandwidth is from $3.1-11.2 \mathrm{GHz}$ with the core of the band always better than $-13.5 \mathrm{~dB}$.

Fig. 5 shows a contour plot of the measured radiation pattern in the $\mathrm{x}-\mathrm{y}$ plane. The plot shows a frequency range from $2-18 \mathrm{GHz}$ versus azimuth angle $(\varphi)$. The antenna is found to have an omnidirectional radiation characteristic throughout the UWB band.

The contour plot radiation pattern for the $x-z$ plane is shown in Fig. 6. Elevation angle $(\theta)$ is plotted versus the frequency range of $2-18 \mathrm{GHz}$. The plots shows typical nulls at $\theta=0^{\circ}$ and $\theta=180^{\circ}$.

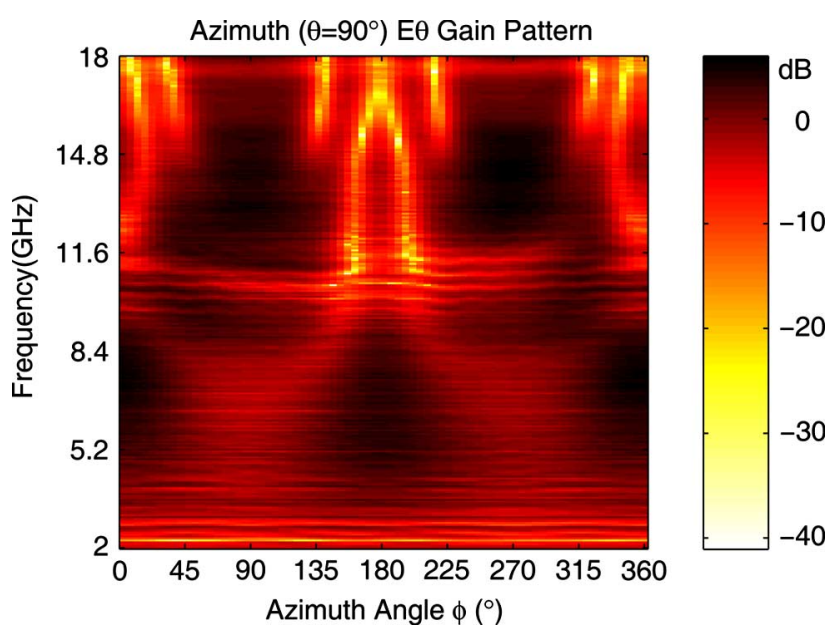

Fig. 5. Measured H-plane radiation pattern for the optimized antenna.

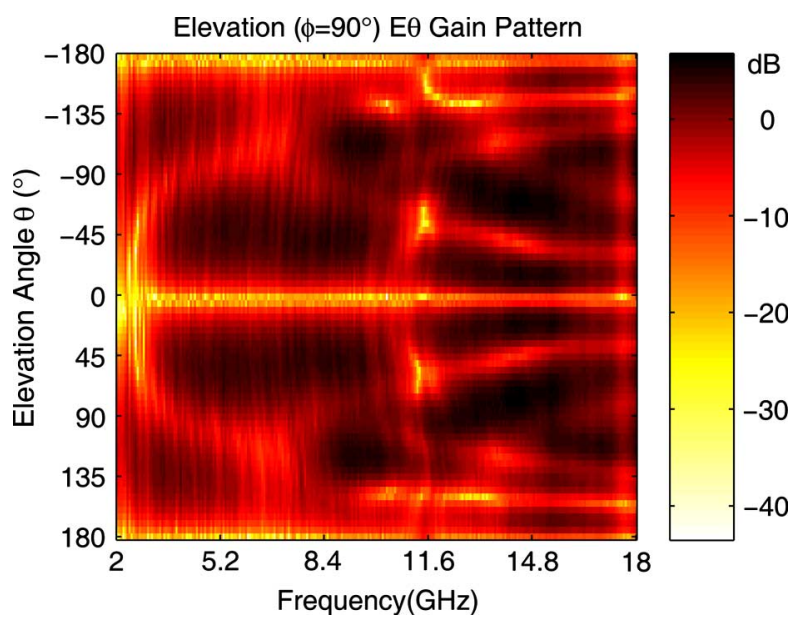

Fig. 6. Measured E-plane radiation pattern for the optimized antenna.

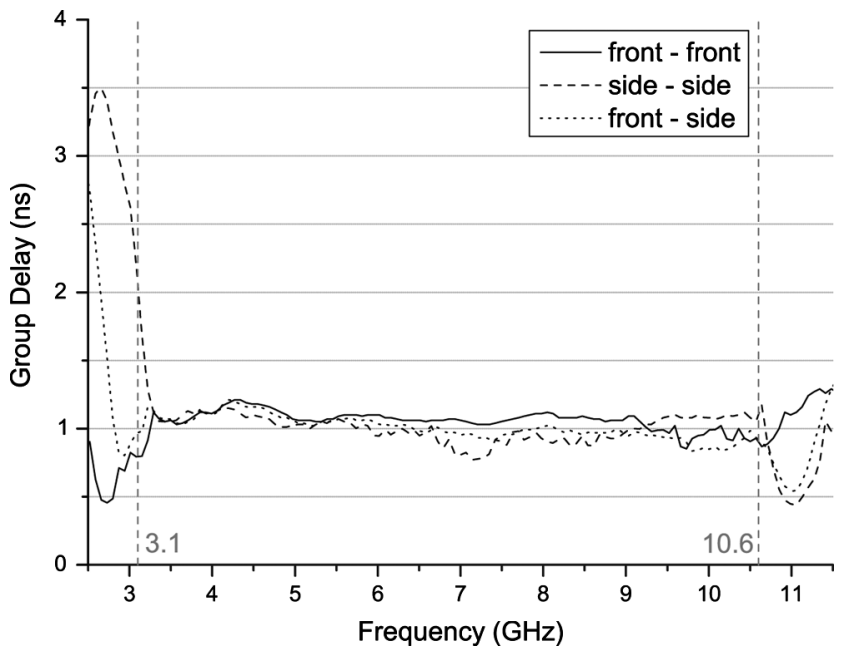

Fig. 7. Group delay between two proposed antennas in different orientations.

Measured group delay of the antenna is shown in Fig. 7. Group delay was measured with a pair of antennas in three orientations, front-tofront, side-by-side and front-to-side. The distance between the antennas is $200 \mathrm{~mm}$. It can be seen that in the UWB group delay variations are within $0.5 \mathrm{~ns}$ regardless of orientation of the antennas. 


\section{CONCLUSION}

We conclude that the combination of a spline based geometry and the multiobjective ParEGO algorithm are suitable tools to optimize a UWB antenna. The antenna geometry as well as the groundplane shape were based on spline curves. Both shapes were optimized simultaneously. The UWB antenna generated by the algorithm shows good radiation patterns and satisfactory group delay. Its small size makes it easy to integrate in UWB devices. ParEGO is a very powerful algorithm to optimize antenna designs, especially if computational resources are limited and only one license for commercial electromagnetic code is available.

\section{REFERENCES}

[1] J. M. Johnson and Y. Rahmat-Samii, "Genetic algorithms in engineering electromagnetics," IEEE Antennas Propag. Mag., vol. 39, no. 4, pp. 7-21, Aug. 1997.

[2] R. L. Haupt, "Antenna design with a mixed integer genetic algorithm," IEEE Trans. Antennas Propag., vol. 55, no. 3, pp. 577-582, Mar. 2007.

[3] A. J. Kerkhoff and H. Ling, "Design of a band-notched planar monopole antenna using genetic algorithm optimization," IEEE Trans. Antennas Propag., vol. 55, no. 3, pp. 604-610, Mar. 2007.

[4] M. F. Pantoja, P. Meincke, and A. R. Bretones, "A hybrid geneticalgorithm space-mapping tool for the optimization of antennas," IEEE Trans. Antennas Propag., vol. 55, no. 3, pp. 777-781, Mar. 2007.

[5] M. John and M. J. Ammann, "Wideband printed monopole design using a genetic algorithm," IEEE Antennas Wireless Propag. Lett., vol. 6, pp. 447-449, 2007.

[6] N. Jin and Y. Rahmat-Samii, "Parallel particle swarm optimization and finite- difference time-domain (PSO/FDTD) algorithm for multiband and wide-band patch antenna designs," IEEE Trans. Antennas Propag., vol. 53, no. 11 , pp. 3459-3468, Nov. 2005.

[7] M. Donelli and A. Massa, "Computational approach based on a particle swarm optimizer for microwave imaging of two-dimensional dielectric scatterers," IEEE Trans. Microw. Theory Tech., vol. 53, pp. 1761-1776, May 2005

[8] L. Lizzi, F. Viani, R. Azaro, and A. Massa, "Optimization of a splineshaped UWB antenna by PSO," IEEE Antennas Wireless Propag. Lett., vol. 6, pp. 182-185, 2007.

[9] J. Knowles, "ParEGO: A hybrid algorithm with on-line landscape approximation for expensive multiobjective optimization problems," IEEE Trans. Evol. Comput., vol. 10, pp. 50-66, Feb. 2005.

[10] M. John and M. J. Ammann, "Spline based geometry for printed monopole antennas," Electron. Lett., vol. 43, no. 6, pp. 317-319, Mar. 2007.

\section{Impedance Matched Ferrite Layers as Ground Plane Treatments to Improve Antenna Wide-Band Performance}

\author{
Faruk Erkmen, Chi-Chih Chen, and John L. Volakis
}

\begin{abstract}
In low-profile applications, ground plane backing significantly degrades antenna performance when the electrical height is a small fraction of a wavelength. An approach to suppress the destructive interference from the ground plane reflections is to employ absorbing layers of ferrite materials. In this paper, we introduce a means to also control and utilize the mode excited in these finite ferrite layers to recover antenna performance at ultra/very high frequencies. Experimental verification is provided using commercial ferrite materials.
\end{abstract}

Index Terms-Absorbing media, ground plane, ultrawideband antennas.

\section{INTRODUCTION}

Wideband conformal antennas are attractive and necessary for airborne and ground communications. However, at low frequencies, keeping the overall antenna height small is a major challenge [1]. A variety of ground plane treatments have been proposed to address this issue, each having different limitations. Certainly, using perfect electric conductor (PEC) is impractical for wide-band low-frequency applications. This is demonstrated in Fig. 1, where we show the gain of a 6-in dipole placed 1 in above a ground plane with various treatments. As shown, the presence of the PEC ground plane resulted in significant gain loss, but use of a matched impedance layer (MIL) on PEC recovers most of the free space gain and even improves it at some frequencies.

Electromagnetic bandgap (EBG) structures and artificial magnetic conductors (AMCs) are other alternatives to overcome the ground plane issue [2]-[4]. Of course, these engineered structures have their own limitations [4]-[8]. Another approach for improving unidirectional radiation is to use absorbing material layers between the antenna and PEC surface [4], [7], [8]. A ferrite layer with possibly $\epsilon_{r} \approx \mu_{r}$ would serve well as a coating to the PEC ground plane and absorb back radiation. Such a layer would provide good matching to incoming waves over a large bandwidth, offering a profile reduction for any antenna class. However, material loss is necessary to suppress destructive interference with antenna radiation, introducing a tradeoff between efficiency and wide-band performance. As ferrite materials are now commercially available, in this paper we investigate their impact on antenna performance. We shall refer to these layers as MILs. Below we consider their effect on antenna impedance and radiation performance as a function of their thickness, dielectric constant, and loss tangent. It is shown that the layer supports a propagation mode that enhances lower frequency performance of the conformally placed antenna. Below, we begin by evaluating the effect of the matched impedance layers over a range of $\epsilon_{r}, \mu_{r}$, and loss tangent values. Then, we focus on commercially available MILs for measurement and concept validations.

Manuscript received March 06, 2008; revised September 13, 2008. Current version published March 04, 2009.

The authors are with the ElectroScience Laboratory, Department of Electrical and Computer Engineering, The Ohio State University, Columbus, OH 43212 USA (e-mail: erkmen.2@osu.edu; chen.118@osu.edu; volakis.1@ osu.edu).

Color versions of one or more of the figures in this paper are available online at http://ieeexplore.ieee.org.

Digital Object Identifier 10.1109/TAP.2008.2009777 